\title{
Chapter 14 \\ Virus Morphogenesis in the Cell: Methods and Observations
}

\author{
Cristina Risco and Isabel Fernández de Castro
}

\begin{abstract}
Viruses carry out many of their activities inside cells, where they synthesise proteins that are not incorporated into viral particles. Some of these proteins trigger signals to kidnap cell organelles and factors which will form a new macro-structure, the virus factory, that acts as a physical scaffold for viral replication and assembly. We are only beginning to envisage the extraordinary complexity of these interactions, whose characterisation is a clear experimental challenge for which we now have powerful tools. Conventional study of infection kinetics using virology, biochemistry and cell biology methods can be followed by genome-scale screening and global proteomics. These are important new technologies with which we can identify the cell factors used by viruses at different stages in their life cycle. Light microscopy, electron microscopy and electron tomography, together with labelling methods for molecular mapping in situ, show immature viral intermediates, mature virions and recruited cell elements in their natural environment. This chapter describes how these methods are being used to understand the cell biology of viral morphogenesis and suggests what they might achieve in the near future.
\end{abstract}

Keywords Virus factory • Virus assembly • Virus morphogenesis • Genomics - Proteomics • Light microscopy $\bullet$ Electron microscopy $\bullet$ Tomography $\bullet$ 3D electron microscopy $\bullet$ Super-resolution microscopy $\bullet$ Correlative microscopy $\bullet$ Molecular mapping • Immunofluorescence • Immuno gold • Immuno electron microscopy - Clonable tags

C. Risco $(\bowtie) \cdot$ I. Fernández de Castro

Cell Structure Laboratory, Department of Macromolecular Structure, Centro Nacional de Biotecnología (CSIC), c/Darwin 3, Campus de Cantoblanco, 28049 Madrid, Spain

e-mail: crisco@cnb.csic.es 


\author{
Abbreviations \\ 2D Two-dimensional \\ 3D Three-dimensional \\ ASFV African swine fever virus \\ CLEM Correlative light and electron microscopy \\ DNA Deoxyribonucleic acid \\ ESCRT Endosomal sorting complex required for transport \\ ET Electron tomography \\ GFP Green fluorescent protein \\ $\mathrm{HCV} \quad$ Hepatitis $\mathrm{C}$ virus \\ HIV Human immunodeficiency virus \\ IEM Immunoelectron microscopy \\ IF Immunofluorescence \\ LM Light microscopy \\ RC Replication complex \\ RNA Ribonucleic acid \\ SEM Scanning electron microscopy \\ TEM Transmission electron microscopy \\ VV Vaccinia virus \\ Y2H Yeast two-hybrid
}

\title{
14.1 Introduction: Cell Biology of Virus Morphogenesis and the Concept of the Virus Factory
}

The idea of viruses as inert molecular entities has progressively been transformed since scientists began to discover the myriad of interactions that occur during the intracellular phase of virus life. Restricted by their own limited genetic repertoire, viruses need to use a number of cell factors for genome replication and morphogenesis. Identification of these factors is essential for understanding the virus morphogenetic processes that often take place in intracellular structures known as viral inclusions, virosomes or viral factories. Viruses are generally thought to build these structures to recruit and concentrate viral and cell factors needed for replication and assembly; they are able to modify a variety of cell organelles and to create new inter-organelle contacts (Fig. 14.1). In the mammalian cell, virus assembly can start inside the nucleus, in association with components of the secretory pathway (Golgi, endoplasmic reticulum or ER), at different points in the endocytic pathway (endosomes, multivesicular bodies or MVB) or at the plasma membrane. Mitochondria and cytoskeletal elements are present in the factories built by many different viruses. 


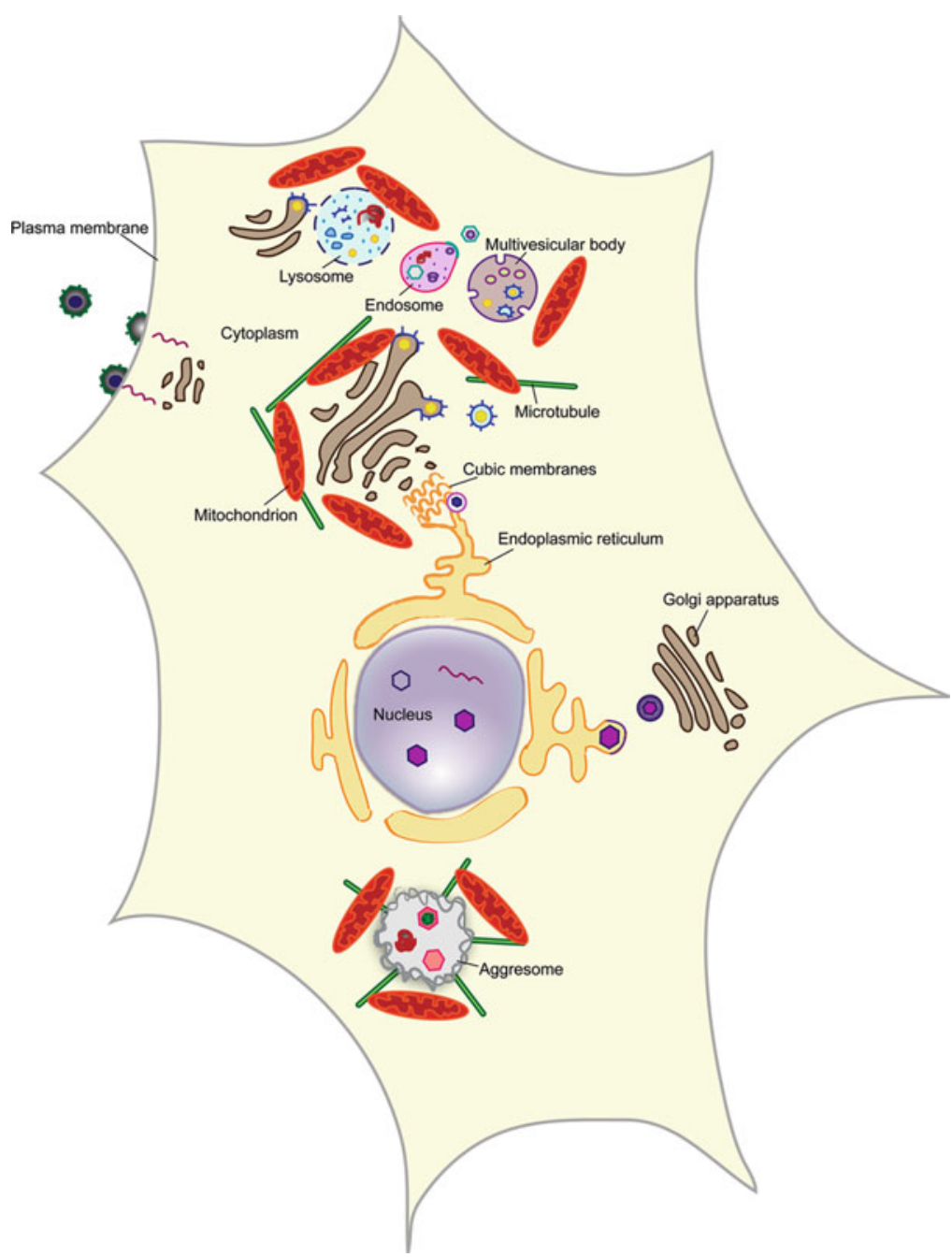

Fig. 14.1 Cell organelles used for viral morphogenesis. The cell nucleus is used by herpes- and papillomaviruses; corona-, bunya- and flaviviruses use components of the secretory pathway such as the endoplasmic reticulum and Golgi; togaviruses and the human cytomegalovirus use components of the endo-lysosomal pathway such as endosomes, multivesicular bodies and lysosomes; the African swine fever virus (ASFV) and poxviruses assemble in aggresome-like structures; assembly sites of retroviruses at the plasma membrane might be connected with a cytoplasmic factory. Viruses modify endomembranes, recruit mitochondria and cytoskeleton, and create new inter-organelle contacts

Virus factories are usually rather large, several microns in diameter, and are very dynamic, as their structure changes over time in accordance with virus needs [1]. This strategy appears to allow viruses to generate progeny with maximum efficiency in the use of cell resources. It is also thought that viruses can use these 
scaffolds to protect themselves from cellular antiviral defences [2]. Viral factories were first described for large DNA viruses such as the African swine fever virus (ASFV) and the poxviruses; we now know that many DNA and RNA viruses build factories in the cytosol, in the nucleus, or both. Due to our limited knowledge of cell nucleus organisation, characterisation of nuclear viral factories is only beginning. In contrast, viral factories assembled in the cytosol are being studied extensively and there are many reports on their architecture and activities. Inside these cytosolic factories, morphogenesis of new viruses begins with the transport of replicated genomes from the structures that harbour the replication complexes (RC) to the assembly sites. Depending on the virus, a number of maturation steps will follow (see Chap. 13 for details) before new viral particles are ready for delivery and propagation.

This chapter will describe fundamental tools for diving inside the infected cell and understanding virus assembly. We also describe relevant examples of virus-cell interactions during virus morphogenesis that are being discovered using in situ techniques.

\subsection{Methods for the Study of Virus-Cell Interactions During Morphogenesis}

By identifying essential cell factors, studies based on recent advances in molecular biology, genomics and proteomics are broadening our knowledge of viral morphogenesis. To confirm and understand the role of potentially interesting genes in viral assembly, the information acquired must be subsequently analysed at a more complex level in infected cells. Exploring existing databases on cell pathways which combine information on genetic, metabolic and signal networks based on the literature can be a first step in further work that on many occasions will also include imaging with a variety of microscopy technologies.

\subsubsection{Identification of Essential Cell Factors}

Characterisation of infection kinetics is the first step in the study of viral morphogenesis. Conventional virology, biochemistry and cell biology methods allow us to determine optimal experimental conditions, including times post-infection (p.i.) and the most appropriate cell types. Key factors can be identified using two main groups of methods, (1) those that analyze gene expression patterns, and (2) those that study protein-protein interactions.

A number of new techniques can be applied to study interactions between viruses and cells on a genomic scale [3]. Microarrays are being used to identify mRNA transcription patterns in different phases of the virus life cycle. DNA gene 
chips detect differences in gene expression between uninfected and virus-infected cells and at different infection stages. Gene data bases are then useful for associating the genes identified with specific cell pathways [4, 5]. High-throughput screening based on RNA interference (RNAi) is another category of methods that analyse gene expression patterns. RNAi is an RNA-dependent gene silencing process within living cells that is often exploited to study the function of genes. This emerging technology is used to study how viruses interact with their hosts at the molecular level. Analysis at various times post-infection has identified a number of cell factors potentially involved in viral morphogenesis, for example for dengue [6], influenza [7] and retroviruses [8]. RNAi may be used for large-scale screens that systematically shut down each gene in the cell. Studies using this approach have shown a requirement for cell factors such as the ESCRT machinery for assembly of the human immunodeficiency virus, HIV [9].

The group of techniques termed proteomics includes powerful methods to study protein-protein interactions. The yeast two-hybrid $(\mathrm{Y} 2 \mathrm{H})$ assay system remains one of the most amenable techniques and is widely used to search for virus-host interactions. $\mathrm{Y} 2 \mathrm{H}$ works by expressing two candidate proteins in the yeast cell. Bait and prey proteins are fused either to a promoter-specific DNA-binding domain or to a transcription activation domain. Interaction between the two proteins in the yeast nucleus brings both domains together so that they can initiate expression of a reporter gene [10]. Individual bait proteins can be screened for interaction with a library of prey proteins. Genome-scale Y2H studies were used to identify 314 virushost interactions for HCV [11] 109 interactions for vaccinia virus [12] and nine for HIV-1 [13]. Similar techniques, such as the yeast three-hybrid system, can be used to study interactions between nucleic acids and proteins [14, 15]. This group of methods also includes pull-down and immunoprecipitation assays, and tandem affinity purification (TAP) tagging approaches, as well as protein identification by quantitative and semi-quantitative mass spectrometry. Whereas $\mathrm{Y} 2 \mathrm{H}$ usually detects transient interactions, affinity-tag purification mass spectrometry shows stable, stoichiometric complexes. Since cell proteins often incorporate into viral particles, these techniques can be applied to the study of protein-protein interactions in the infected cell and in purified viral assembly intermediates [16]. This is the case of clathrin, for example, which was found in retrovirus particles; clathrin was only recently identified as one of the cell factors that facilitate accurate morphogenesis of several retroviruses [17]. Y2H technology also detected cell proteins that interfere with virus assembly and viral proteins that block them. This is the case of tetherin, first detected by proteomics and mass spectrometric protein identification as a cell factor that restricts retrovirus assembly [18], and later confirmed as a restriction factor for a wide variety of enveloped viruses [19]. Viruses have several anti-tetherin proteins to counteract the effect of this factor [20]. The current challenge of high-throughput technologies is to develop more efficient informatics tools to accurately analyse the vast amount of information they can provide [21].

The next step in the characterisation of in-cell virus assembly pathways would be to study the specific roles of the factors identified in their natural environment; to 
do this, we must of course return to the infected cell. Functions can be tested by protein depletion or overexpression, by mutagenesis or by protein targeting with tags. To visualise key factors in infected cells, there are a variety of classical and novel microscopy techniques that will be described in the following sections.

\subsubsection{Studying Viral Morphogenesis in Situ with Light and Electron Microscopy}

Microscopy has played an essential role in our understanding of cell architecture and viral assembly (see Chap. 3). Light microscopy (LM) and transmission electron microscopy (TEM) provide different types of information about viral infection, ranging from general events that involve the whole cell to the detailed imaging of nascent and maturing viral particles in specific cell compartments [22] (Fig. 14.2). With resolutions in the 100-500 $\mathrm{nm}$ range, LM shows organelle recruitment and modification in the assembly compartment and, in the case of the largest viruses, individual new viral particles as well [23]. In immunofluorescence assays using antibodies to viral proteins and cell compartments, we can see where viral structural and scaffolding proteins (Chap. 11) concentrate to create the assembly sites. Light microscopy shows, for example, that the same virus can build distinct factories in different cell types, depending on specific characteristics of the cell (Fig. 14.2a, b). Functional viruses that express proteins fused with clonable fluorescent tags such as the green fluorescent protein (GFP) (see Sect. 14.3 for details) can be followed in live cells [24]. Video microscopy facilitates dynamic characterisation of the biogenesis of the factory and virus assembly in real time. Time course experiments with antibody-labelled permeabilised cells or video microscopy studies with GFP fusions in living cells are essential for selecting specific conditions, such as the best times p.i., for more detailed, higher resolution study by TEM.

Electron microscopy generally uses ultra-thin sections of cells previously embedded in plastic resins after conventional fixation and dehydration; alternatively, cells are processed at low temperature after or upon fixation for optimal preservation of ultrastructure (see Chap. 3 for sample preparation details), or subjected to criofixation procedures such as high-pressure freezing prior to freeze substitution and embedding, or freeze-fracture analyses. With resolutions in the range of a few nanometers, cell TEM can show changes in shape and size of virus assembly intermediates in specific intracellular compartments [25, 26] (Fig. 14.2c, d). TEM of infected cells shows that mitochondria, endomembranes and cytoskeleton often participate in organising the structure that supports viral assembly. To complete maturation and become infectious, immature viral particles must often travel within the factory to find specific cell factors. At late times post-infection, once virus progeny have been produced and must find their way out of the cell, the factory can be dismantled [1]. 

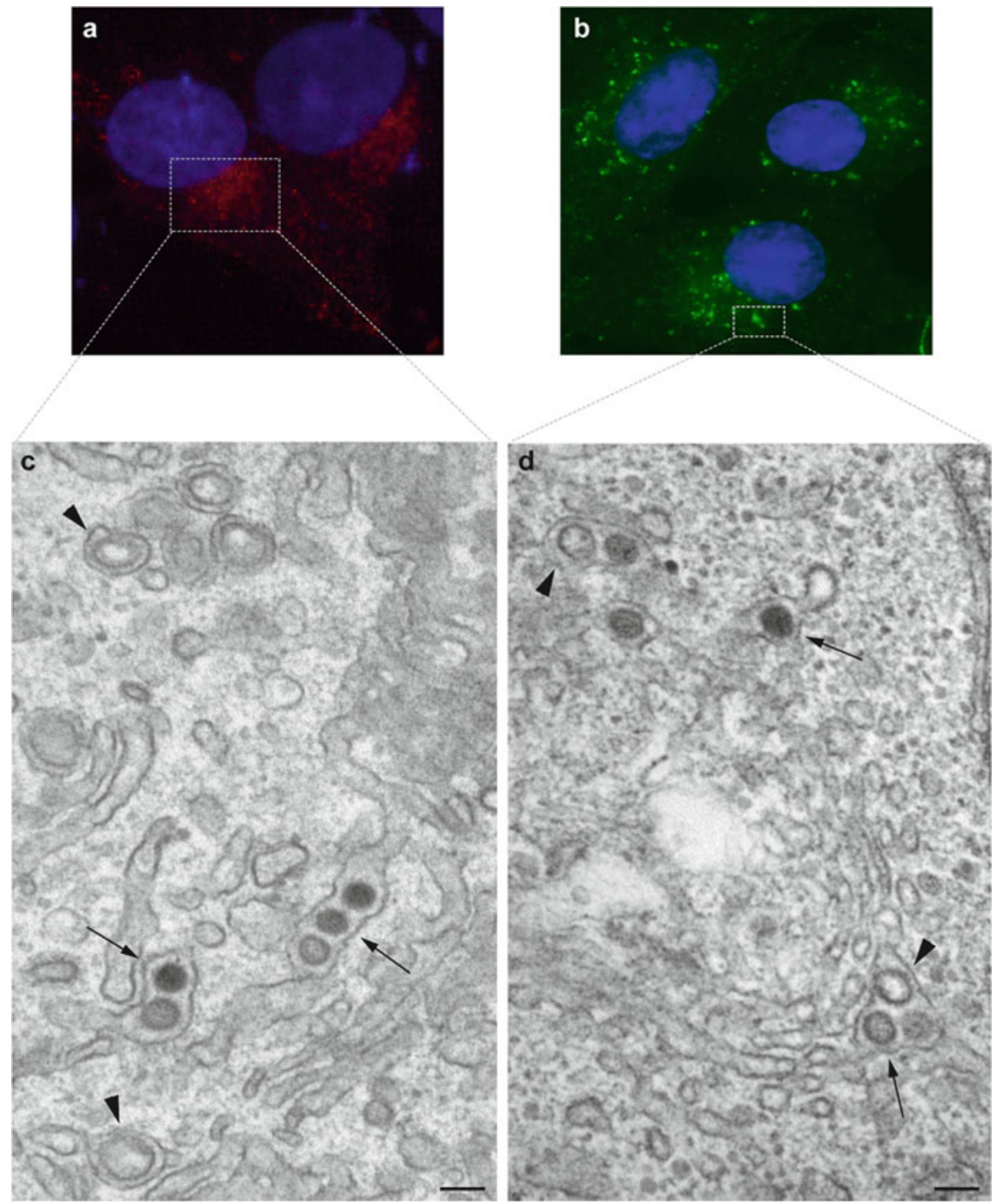

Fig. 14.2 Studying virus assembly by light and electron microscopy. (a, b) Immunofluorescence microscopy of BHK-21 (a) and Vero cells (b) infected with a bunyavirus at $10 \mathrm{~h}$ p.i. (h.p.i.). Cells were labelled with an antibody specific for one of the viral structural proteins that concentrates at the assembly sites. A single large perinuclear factory is formed in BHK-21 cells, whereas many mini-factories are seen in Vero cells. (c, d) TEM of BHK-21 (c) and Vero cells (d) at 10 h.p.i. In both cases, similar spherules, the structures that harbour the RC (arrowheads) [22] and viral intermediates (arrows) are distinguished in Golgi membranes. Scale bars, $100 \mathrm{~nm}$

TEM of cells in two dimensions provides a first glimpse of the viral morphogenetic pathways in situ. The size of cells (several microns in diameter) and the thickness of ultra-thin sections (50-100 nm) nonetheless constitute a major 
limitation, because we are actually studying single planes of much larger structures. In these conditions, many elements and contacts can be missed. Three-dimensional TEM avoids this limitation; the variety of methods available is described in the following sections.

\subsubsection{Visualising Virus Assembly in Three Dimensions I: 3D Reconstructions of Serial Sections, TEM and SEM}

In conventional TEM, electrons must traverse the sample to generate a projection image. Samples must therefore be thin, around $50-100 \mathrm{~nm}$, thinner than many viruses. Given that eukaryotic cells are several microns in diameter, the ultra-thin sections are single planes of much larger structures. For conventional TEM, cells are fixed on culture plates and collected by low speed centrifugation, followed by sectioning of the pellet. In these conditions, cells preserve their morphology but present a variety of orientations (Fig. 14.3a); analysis is therefore restricted to random, unique sections of cells, and scarce or non-randomly distributed elements can be missed completely. Oriented serial sections solve this problem (Fig. 14.3b). By collecting all serial sections from each cell, all intracellular elements can be detected and studied. This strategy guarantees a complete analysis without missing any intracellular event of interest; immunofluorescence can assist by localising where viruses are assembled within a cell and thus, where cells should be sectioned for TEM [22]. The study of oriented serial sections is very informative and has revealed unreported contacts between cell organelles, $\mathrm{RC}$ and assembly sites, as well as the relationships of different viral intermediates with specific cell elements (Fig. 14.3c). After image processing and segmentation to assign identities to all individual structures in the images, serial sections can be aligned and combined in 3D reconstructions [27]. With resolution values of $\sim 5 \mathrm{~nm}$ in the $\mathrm{X}$ and $\mathrm{Y}$ axes, these 3D reconstructions can show viruses in factories with considerable detail (Fig. 14.3d, e). Resolution in the $\mathrm{Z}$ axis is limited, however, with values of $\sim 50-100 \mathrm{~nm}$ due to imperfections in the alignment process.

Scanning electron microscopy (SEM) can also be used to visualise virus morphogenesis. These microscopes use a focused beam of high-energy electrons to generate a variety of signals at the surface of solid specimens. Samples are covered by a thin layer of metal and scanned with a primary source of electrons; secondary electrons are then released from the sample surface and collected by a detector. The signals derived from electron-sample interactions furnish information about the sample, including external morphology in 3D. Modern scanning electron microscopes can now provide resolutions as precise as $2-5 \mathrm{~nm}$, near that achieved by cellular electron microscopy [28]. SEM images can show morphological changes in large virus particles during maturation in situ; this is the case, for example, of the giant mimivirus (Fig. 14.4a, b). In lysed cells and isolated virus factories visualised by SEM, immature viruses are clearly distinguished at short 
a

Cell pellets

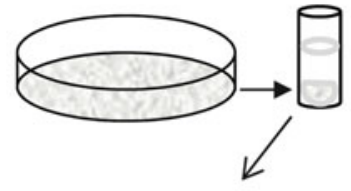

Random sectioning

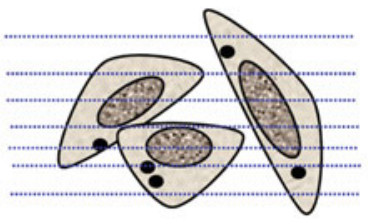

C

\section{TEM}
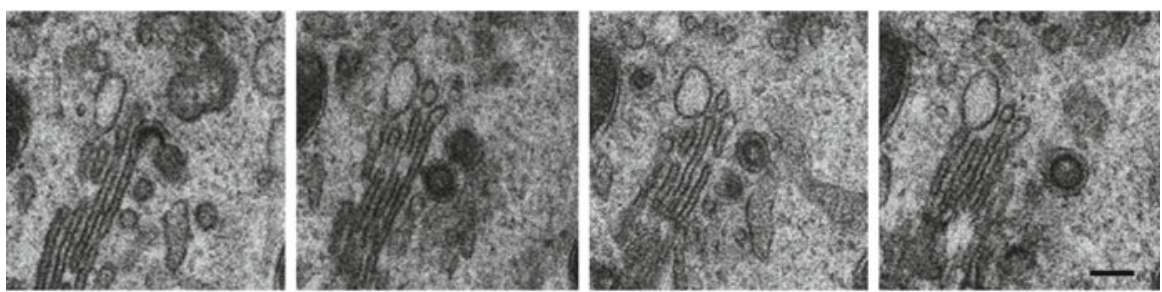

\section{D reconstructions}
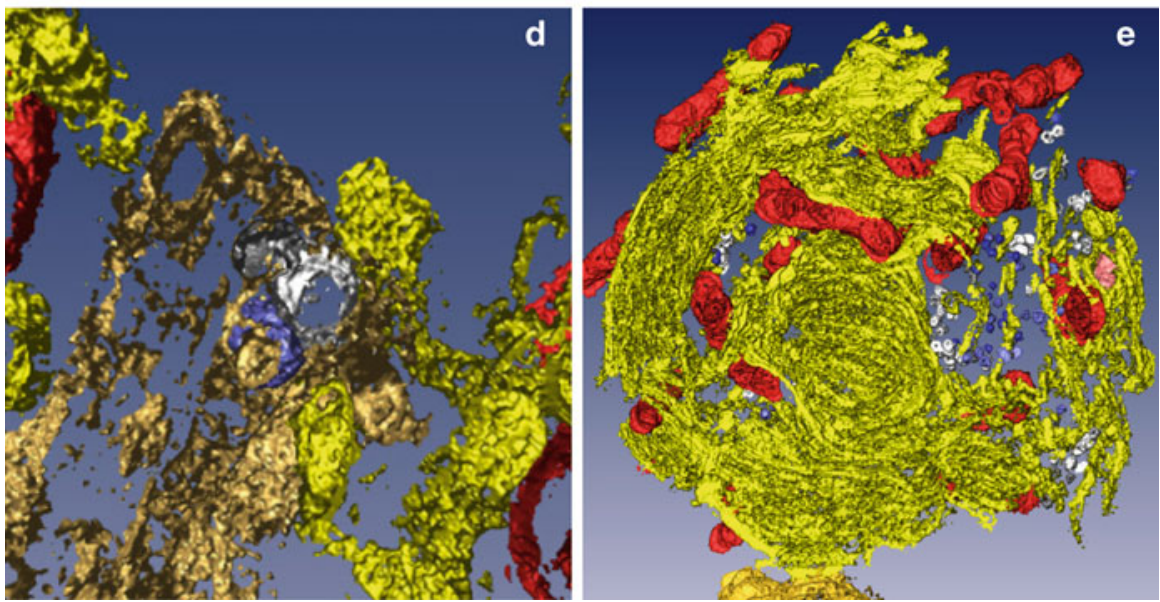

Fig. 14.3 Serial sections, TEM and 3D reconstructions. (a, b) Summary of the principles and differences between conventional ultra-thin sectioning and oriented serial sectioning. (c) Serial sections in TEM and (d) 3D reconstruction showing the interaction between a spherule, the structure that harbours the RC (white) and a viral particle (blue) in Golgi membranes (beige). Mitochondria are segmented in red and rough endoplasmic reticulum (RER) in yellow. (e) 3D reconstruction of a viral factory from a different cell. In this case, 15 serial sections were used. The Golgi complex has been removed to improve visualisation of RC and viral particles. Scale bar, $100 \mathrm{~nm}$ 

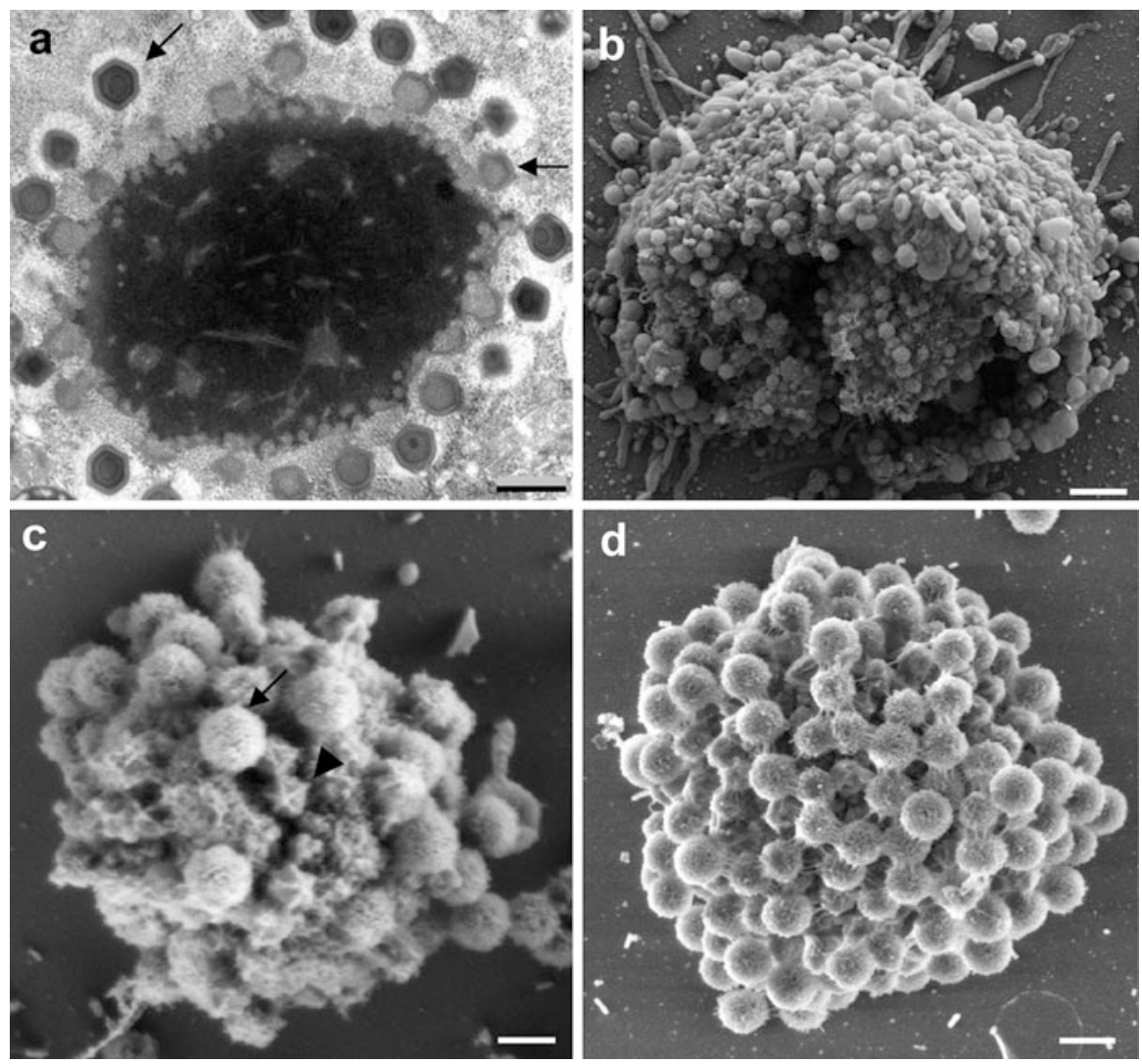

Fig. 14.4 Mimivirus factory in 2D and 3D by TEM and SEM. (a) 2D views of a virus factory as visualised by TEM, showing virus particles (arrows) at various assembly stages. (b) 3D views of a viral factory within an amoeba cell lysed at 8 h.p.i. and visualised by SEM. (c) SEM of a factory isolated at 8 h.p.i. Viral particles are seen at various assembly stages. The arrow indicates a mature virus particle and the arrowhead, an immature particle. (d) SEM of a virus factory isolated at $10 \mathrm{~h}$. p.i. Only mature viruses can be detected. Scale bars, $500 \mathrm{~nm}$ in (a) and (d); $2 \mu \mathrm{m}$ in (b); $300 \mathrm{~nm}$ in (c) (Reproduced with permission from [29])

times p.i. (Fig. 14.4c), whereas mature virions accumulate in the factory at longer times p.i. (Fig. 14.4d) [29].

TEM in combination with freeze-fracture and metal shadowing allows higher resolution $3 \mathrm{D}$ views of viruses inside intact cells. Frozen cells are fractured mechanically and covered with a thin layer of platinum and carbon in a vacuum chamber, the metal replicas are then washed to eliminate organic materials and mounted on EM grids. The main limitation of this approach derives from the unpredictable patterns of the fracture planes and the difficulty in interpreting the images. It can nevertheless be useful when studying the morphogenesis of large enveloped viruses (Chap. 11) and their interactions with cell endomembranes during assembly and maturation [30]. 


\subsubsection{Visualizing Virus Assembly in Three Dimensions II: Electron Tomography}

Electron tomography (ET) of infected cells shows virus assembly in 3D with resolutions of $\sim 3-5 \mathrm{~nm}$ in all three axes, $\mathrm{X}, \mathrm{Y}$, and $\mathrm{Z}$ (see Chap. 3). Higher resolutions of $\sim 1 \mathrm{~nm}$ have been reported, as was the case of the budding HIV viral particles visualised by cryo-electron microscopy on the surface of intact human cells [31]. Both normal and aberrant budding events were visualised on the cell surface, suggesting that cellular and/or viral factors control the quality of virus assembly and maturation. A limitation of cellular tomography is that the maximum thickness of the sample must be $<0.5 \mu \mathrm{m}$, whereas virus factories are larger; however, conventional TEM methods like those described above can help to select specific elements within cells for more detailed ET structural analysis. Thanks to ET, elusive structures have been visualised for the first time, such as the transfer of viral genomes from RC to assembly sites in dengue virus-infected cells [32] (Fig. 14.5a, b). The morphogenesis of poxviruses is another good example; vaccinia virus (VV) assembly and architecture were the subject of numerous studies, but the organisation and biogenesis of immature and mature VV particles were not understood until the first ET studies were carried out [33,34]. These analyses showed unprecedented remodelling of cell endomembranes during VV particle assembly (Fig. 14.5c, d). Tomograms are analysed in detail in computational slices of 1-2 nm extracted from the original volumes (Fig. 14.5a). As with serial sections in conventional TEM, the relevant information is contained in these single planes, whereas 3D representations are models used to summarise the most relevant features in the tomograms (see Chap. 3 for details).

In summary, two main groups of methods are available for 3D studies of viral assembly. 3D reconstructions of serial sections by TEM or analysis of surface morphology by SEM will show the general organisation of the virus factory, the inter-organelle contacts, and changes in the cell compartments where viruses are formed. In contrast, electron tomography is more appropriate for studying individual viral particles and to obtain fine details of their maturation in situ.

\subsection{Molecular Mapping of Viral Morphogenesis}

The discipline known as histochemistry includes a great variety of techniques to visualise molecules in biological samples [35]. There are several histochemical methods to localise nucleic acids, lipids, sugars, and other molecules, some of which have been used to label viruses in cells. Nonetheless, protein-labelling techniques are by far the most developed; because of their importance in the study of virus morphogenesis, in this section we will focus on methods that detect proteins in light and electron microscopy. 

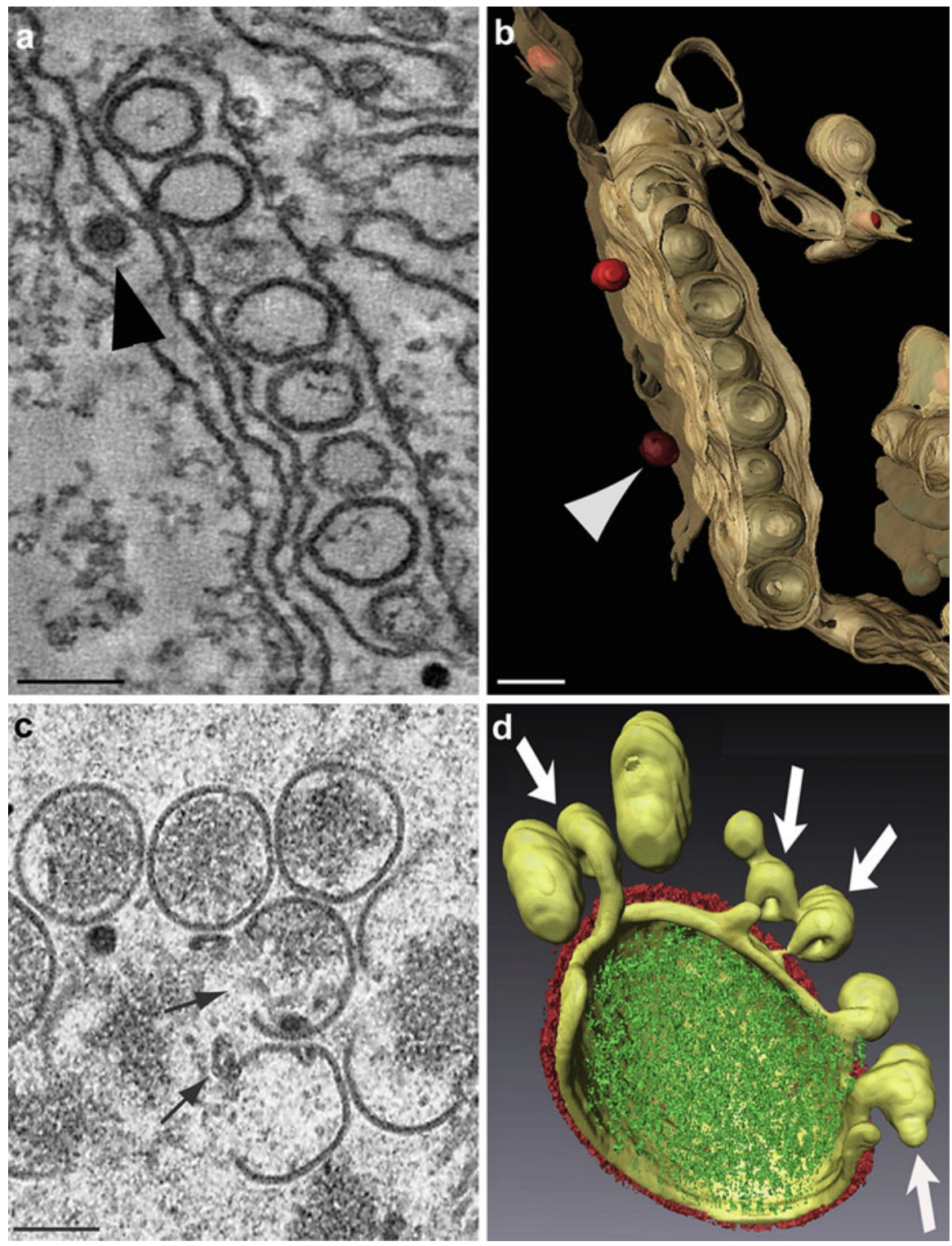

Fig. 14.5 3D electron tomography of virus assembly. (a) Computational slice and (b) 3D reconstruction of the dengue virus factory as visualised by ET. Nascent viral particles (arrowheads) face the spherules that harbour the RC. (c, d) 2D TEM and 3D ET, respectively, of immature VV particles in the process of assembly from cell membranes (arrows). ET shows how the viral envelope is connected to a collection of open membrane structures and how these membranes contribute to envelope formation. Scale bars, $100 \mathrm{~nm}$ in (a) and (b); $250 \mathrm{~nm}$ in (c) (Reproduced with permission from [32] (a) and (b), and [33] (d)) 


\subsubsection{Antibodies, Immunofluorescence and Immunoelectron Microscopy}

Previous sections of this chapter have shown how to identify key factors for virus morphogenesis and how to visualise virus assembly in cells in two and three dimensions. To link the information from both methods and to fully understand viral morphogenesis, we also need methods for molecular mapping in situ. Molecules of interest, which are viral and cellular factors, can be localised in cells with specific antibodies in immunolabelling assays or with clonable tags (Fig. 14.6).

Antibodies detect proteins with high specificity and variable sensitivity. Primary antibodies generated against proteins of interest are detected with secondary antibodies conjugated to a fluorescent probe for LM visualisation, or to an electrondense colloidal gold particle, which is easy to detect by EM in immunogold assays (Fig. 14.6a). Antibodies have been and are still fundamental tools in electron microscopy $[36,37]$. When immunolabelling proteins on cryosections, where cells have not been dehydrated and maintain their proteins in a natural hydrated state, the sensitivity of antibody detection is usually higher than that obtained when labelling sections of dehydrated, resin-embedded cells. Since the introduction of cryosectioning, the method has been improved and perfected [38, 39]. This approach allows colocalisation of nascent and maturing viral particles in specific cell compartments and the proteins being incorporated into assembling virus particles (Fig. 14.6b). Although antibodies are usually very specific, information derived from these experiments is later confirmed in biochemical assays.

Due to their large size, antibodies that recognise internal structures must be used on cell sections. Alternatively, cells can be permeabilised to label intracellular compartments (Fig. 14.6c), although this is incompatible with preservation of fine ultrastructure. Certain permeabilisation protocols use the bacterial exotoxin streptolysin O (SLO) to open pores in the plasma membrane while leaving intracellular membranes untouched. This was used in pre-embedding immunogold assays to follow viral and cell proteins during VV assembly from intracellular membranes [40].

An important limitation of antibodies is their variable sensitivity due to epitope loss during sample preparation and to macromolecular interactions inside cells that often mask the protein epitopes in vivo. This is particularly problematic when the proteins of interest are part of densely packed macromolecular complexes, such as those involved in virus assembly. The use of clonable tags can overcome these limitations.

\subsubsection{Clonable Tags}

Jellyfish green fluorescent protein (GFP), its mutants and homologues have caused a true revolution in cell biology. If proteins fused to a fluorescent tag maintain their 
a
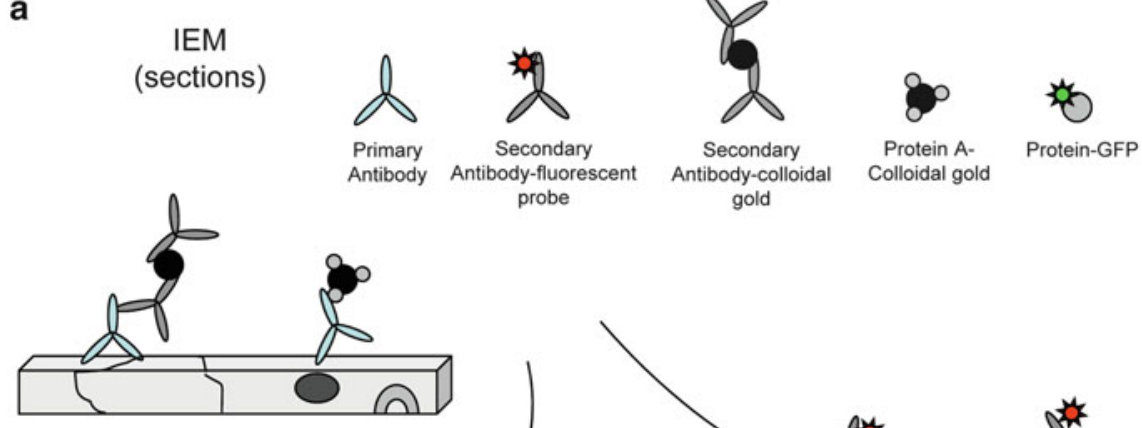

probe

gold
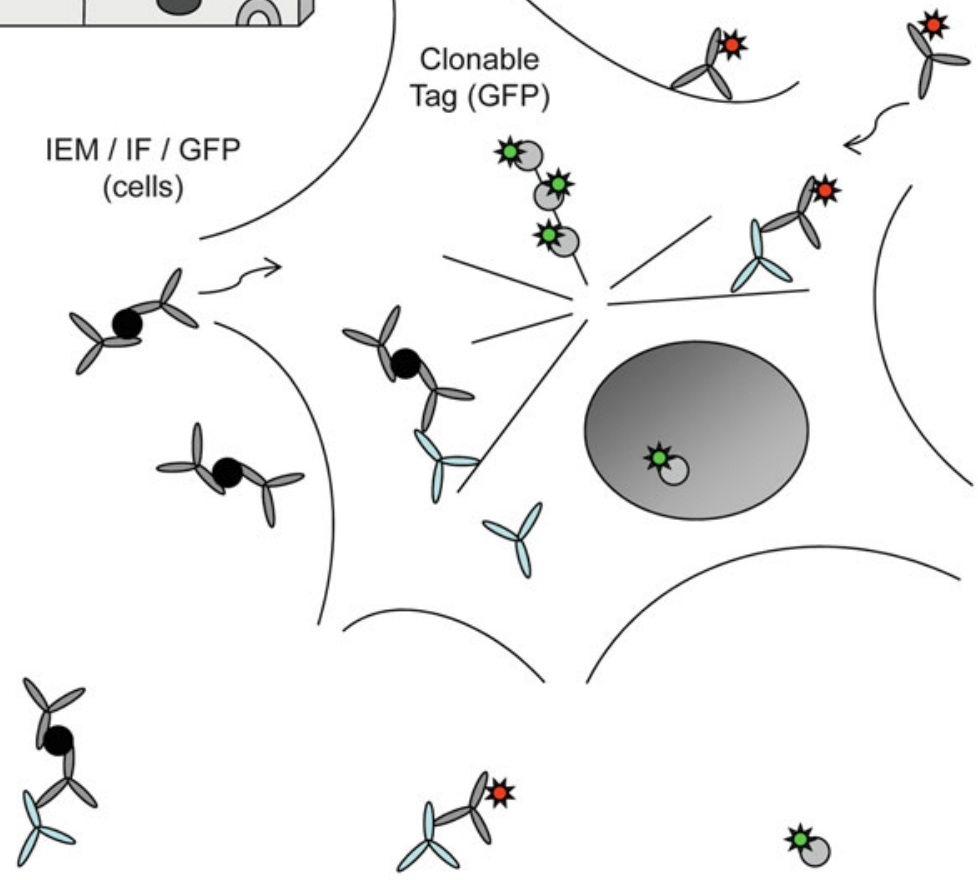

赵

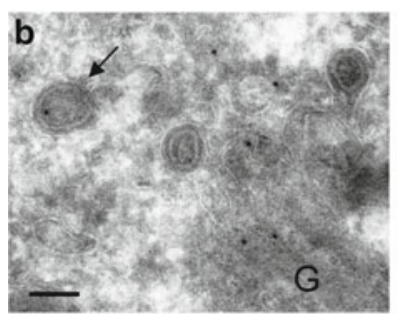

cryosection

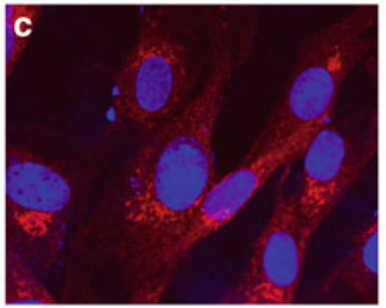

permeabilized cells

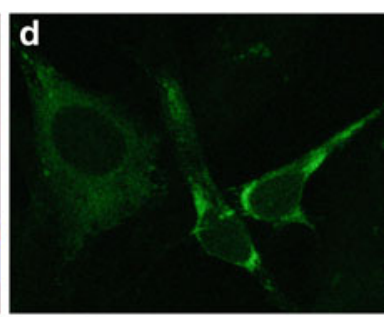

live cells

Fig. 14.6 Molecular mapping of virus assembly with antibodies and clonable tags. (a) Schemes showing the principles of labelling on thin sections, whole permeabilised cells and intact cells. (b) Immunogold detection on a cryosection from a bunyavirus-infected cell. Cells were labelled with a primary antibody specific for a viral scaffolding protein, followed by secondary antibodies conjugated to $10 \mathrm{~nm}$ colloidal gold particles. The protein is detected in Golgi membranes $(\mathrm{G})$ and 
normal functions, their movements and interactions with intracellular structures can be tracked in living cells (Fig. 14.6d). In conjunction with the new generation of LM methods, we can even follow the assembly of individual virus particles at real time [41]. GFP is a relatively large molecule $(27 \mathrm{kDa})$ and, since viral proteins have very strict structural requirements, fusion to GFP can produce loss of function. If this is the case, probes smaller than GFP could be a viable option. The group of smaller probes includes the fluorescent flavoprotein known as miniSOG, which is less than half the size of GFP [42], or the smaller tetra-cysteine motifs, which are visualised after incubation with fluorescent biarsenical molecules such as ReAsH or FlAsH [43].

The use of clonable tags in TEM and ET would be a considerable advance for high resolution studies of virus assembly. The first clonable tags for EM validated in cells have yet to be used in virus morphogenesis studies, but show good prospects and will be listed in Sect. 14.5.3.

\subsection{The Search for Signalling Pathways}

The initiation of virus morphogenesis usually requires major reorganisation of cell membrane compartments and the cytoskeleton. Many laboratories are applying the methods described in previous sections to understand how viruses take control of cells and recruit all the necessary materials for their morphogenesis. The complexity of the interactions inside infected cells can be extraordinary, as illustrated by the viruses known as virophages that parasitise the factory built by other viruses. Some images of cells co-infected by the giant mimivirus and the virophage "Sputnik" suggest that the small virophage not only uses the materials recruited by the mimivirus for its own replication, but might even hide inside the mimivirus particles to exit the cell [44]. We still do not know how organelles and materials are recruited or how replicated viral genomes are transported from $\mathrm{RC}$ to assembly sites. We do know, however, that viruses target their proteins to specific cell compartments, that some viruses use a cellular defence mechanism termed the aggresome response, and that signals related to organelle movement on cytoskeletal tracks could also be involved $[1,2]$.

Fig. 14.6 (continued) viral particles (arrow). (c) Immunofluorescence detection of the same scaffolding protein in permeabilised cells. (d) Still image from a video recorded in a fluorescence microscope equipped for live cell imaging. Cells were infected with a recombinant virus that expresses the same scaffolding protein fused with GFP. Scale bar, $100 \mathrm{~nm}$ 


\subsubsection{DNA Viruses and Cell Aggresomes}

Aggresomes are a defence response of cells to protein misfolding and aggregation. These inclusions form at the microtubule-organising centre (MTOC), where they enclose potentially toxic protein aggregates within vimentin cages. The large DNA viruses of animals such as the African swine fever virus (ASFV), the poxviruses, and the iridovirus frog virus three build factories that closely resemble cell aggresomes (Fig. 14.7a) [1, 2, 45, 46]. Virus factories and aggresomes both assemble at the MTOC, recruit mitochondria and cell chaperones, build a cage with vimentin filaments, and are maintained by the activity of dynein motors on microtubules. EM shows membranes, ribosomes, viral intermediates and fully assembled viruses inside the vimentin cage of the ASFV aggresome-like factory (Fig. 14.7b). It is suggested that viruses kidnap the aggresome pathway to avoid being recognised as foreign, or alternatively, to be mistaken for a misfolded protein by the cell, thus triggering the aggresome response [2].

A similar strategy might be used by viruses that replicate and assemble in the cell nucleus and associate with structures known as POD (potential oncogenic domains). POD are nuclear aggresomes used by herpes-, papilloma-, adeno- and parvoviruses. Recent observations suggest that some RNA viruses use aggresomes to build their factories. Since the common feature of factories built by RNA viruses is the remodelling and recruitment of cell endomembranes, however, different signalling pathways must be triggered in this case.

\subsubsection{RNA Viruses and Membrane Remodelling}

DNA viruses usually build distinct, and even distant factories for genome replication and morphogenesis. Herpesviruses, for example, must connect the first steps of assembly inside the nucleus with subsequent incorporation of proteins and membranes in the cytosol; poxviruses must coordinate replication in cytosolic mini-nuclei with primary assembly in aggresome-like structures and final wrapping in the Golgi apparatus [1]. In contrast, RNA viruses often induce the construction of a single sophisticated membranous web in which replication complexes and assembly sites are located near each other. Expression of viral replicase complexes is usually sufficient to trigger membrane remodelling and organelle recruitment [47, 48].

Viruses take control of cell endomembranes by interfering with lipid metabolism, protein regulation and transport. The secretory pathway is the most common target for this virus-induced membrane remodelling, while the endocytic pathway also participates in some cases (Fig. 14.1). The two pathways are closely related, in fact, and converge at the trans-Golgi compartment. Described in the literature with many different names, the membranous tubuloreticular structures (TBS) often detected in infected cells are indeed cubic membranes that consist of highly curved, 3D-folded lipid bilayers. Alterations in cholesterol metabolism are linked to the 
a

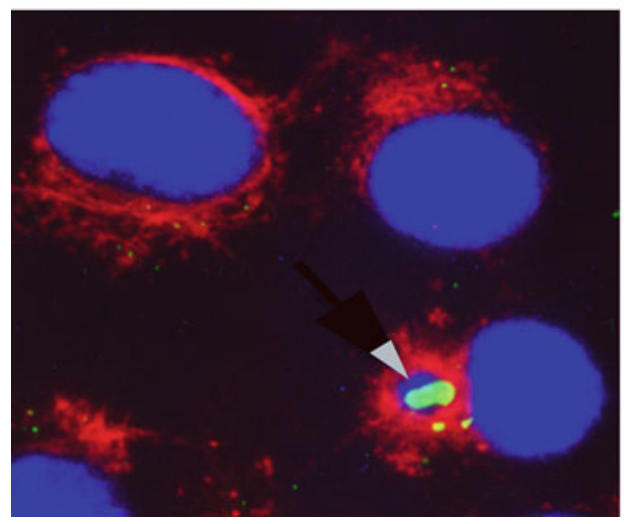

b

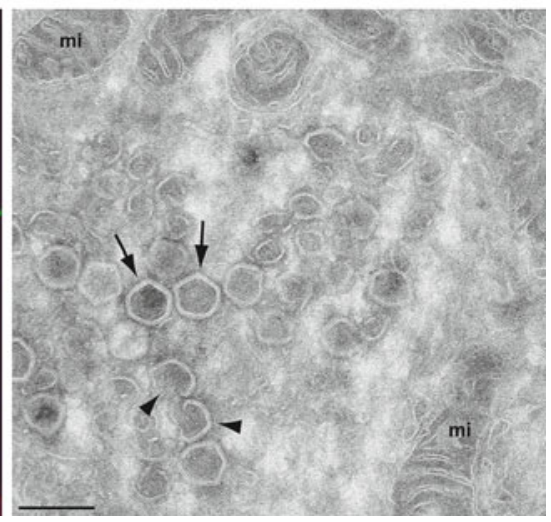

Fig. 14.7 Virus assembly in cell aggresomes. (a) The factories of the ASFV resemble cell aggresomes, as shown by immunofluorescence. Viral proteins (green) co-localise with DNA (blue) inside vimentin cages (red). (b) TEM of an ASFV factory, showing mitochondria (mi), viral intermediates (arrowheads) and mature viruses (arrows). Scale bar, $250 \mathrm{~nm}$. Images kindly provided by Dr. Germán Andrés (CBMSO-CSIC, Spain)

biogenesis of cubic membranes, used for assembly by some viruses such as the SARS (severe acute respiratory syndrome) coronavirus [49]. Other viruses depend on phosphatidylcholine synthesis, fatty acid synthesis or geranylation. The highly curved nature of cubic membranes, possibly induced by multiple interactions between replicase proteins, might assist virus budding. Virus replication often slows secretion, however, which means that viruses can interfere with membrane trafficking proteins and their regulation by small GTPases [2, 47]. Additional host factors involved in virus-induced membrane remodelling include the SNARE (soluble NSF attachment protein receptor) proteins, which are mediators of vesicle fusion used by the human cytomegalovirus (HCMV), the VAMP-associated proteins (VAP) that bind to SNARE and are used by hepatitis C virus, and the ESCRT (endosomal sorting complex required for transport), which are essential for HIV assembly in the plasma membrane [19, 47, 50].

Inside membranous webs, virus assembly is precisely coordinated with transport of replicated viral genomes from the $\mathrm{RC}$; the specific machinery remains to be identified. Lessons from plant viruses and their movement proteins involved in viral genome transport inside cells and between cells through plasmodesmata can provide some clues for the study of animal viruses [51].

Finally, signalling related to cytoskeleton-mediated organelle movement, particularly in the case of mitochondria, can be essential for factory assembly and virus morphogenesis. Mitochondria attach to membranous webs and are thought to provide energy for viral factory activities. They can have additional roles, as in infected cells it was observed that some mitochondrial proteins abandon the organelle and are integrated into the factories, where they interact with viral proteins $[52,53]$. 


\subsection{Perspectives and Conclusions}

Some of the following new methodologies have not yet been applied to the study of viral morphogenesis, but their capacity to track macromolecules in cells suggests great potential for the study of virus assembly.

\subsubsection{Super-Resolution Fluorescence Microscopy}

Limited by the diffraction barrier, conventional light microscopy methods reach resolutions of $\sim 100-500 \mathrm{~nm}$. Many viruses and cell substructures are smaller than this and cannot be solved by LM. In recent years, several laboratories have developed a group of optical microscopy methods, termed super-resolution microscopy, that have improved spatial resolution by an order of magnitude over the diffraction limit [54]. Future improvements in microscopes, fluorescent probes and labelling chemistry will further refine the resolution of these methods, considerably narrowing the gap between light and electron microscopy. Using a variety of technical strategies, these new technologies have begun to provide insights into cell biology and virology. In particular, real-time imaging methods that track individual virus particles in living cells are being used to study virus assembly, and super-resolution microscopy has already defined interactions with cell factors during HIV-1 budding $[55,56]$. In the near future, these microscopies will have an increasing impact in the field of virus morphogenesis.

\subsubsection{Correlative Microscopy: From Live Cells to High Resolution}

Electron microscopy has contributed more than any other method to our understanding of virus assembly in the cell, although its static nature nonetheless makes it difficult to characterise highly dynamic processes. Correlative light and electron microscopy (CLEM) combines the advantages of live cell imaging with the high resolution of EM. A number of procedures have been reported, and the method of choice depends on the research question [37]. Basically, with CLEM we can select individual live cells with interesting features for a detailed, high-resolution study in TEM and ET. Finding adequate probes for CLEM will be the main technical challenge of these studies. 


\subsubsection{Clonable Tags for Electron Microscopy and Tomography}

Genetically clonable tags for TEM and ET would supply new strategies for the ultrastructural characterisation of virus assembly. To date, two types of approaches have been reported, using photoconversion of fluorescent tags or metal-binding proteins. Diaminobenzidine (DAB) can be photoconverted by production of singlet oxygen from fluorescing proteins to generate dense osmiophilic precipitates that are visible in the electron microscope [57]. The resulting signals are diffuse and lack the resolution of particulate probes, although reasonable results have been obtained for proteins concentrated in cell organelles and in electron tomography [37, 42].

Metal-binding proteins were recently validated as clonable tags for EM in bacteria [58, 59] and mammalian cells [60]. These methods allow detection of intracellular proteins at high sensitivity and molecular scale resolution. To track protein molecules in CLEM, proteins can be double-tagged with a metal-binding peptide and a fluorescent probe. This will give us a new way to look inside cells and visualise where and how individual macromolecules come together to build viral particles.

\subsubsection{Conclusions}

New technologies have recently begun to offer access to analysis of viruses in cells in unprecedented detail. The complexity of the interaction networks established in these contexts is changing our concept of viruses from that of inert molecular organisms to "live" entities able to carry out a wide variety of activities inside cells.

The different technologies and their integration for the study of virus-cell interactions are summarised in Fig. 14.8. From the conventional techniques used to characterise infection kinetics to the new developments in proteomics, genomics, bioinformatics and microscopy, research in this field is generating a vast amount of information about how viruses manage to assemble all the machinery needed to build infectious particles from viral macromolecules and cell materials. Relevant examples on what is being learned on the participation of host cell factors in virus assembly have been described in this chapter by focusing on studies with a few representative model systems. Future work must be accompanied by studies of how cells position their proteins and regulate organelle shape, size and movement. By understanding how viruses manipulate these processes, we will not only learn about viruses but also about cell architecture and compartmentalisation of functions.

Acknowledgements Our gratitude to Drs German Andrés, Abraham Minsky, Ralf Bartenschlager, Petr Chlanda, Jacomine Krijnse-Locker, Laura Sanz, José Jesús Fernández, Juan Fontana, and Noelia López Montero for providing images, and to Catherine Mark for editorial assistance. Work in C.R.'s laboratory was funded by a grant from the Ministry of Science and Innovation of Spain (BIO2009-07255). I.F.C. is recipient of a fellowship from the FPI Program of the Spanish Ministry of Economy and Competitiveness. 


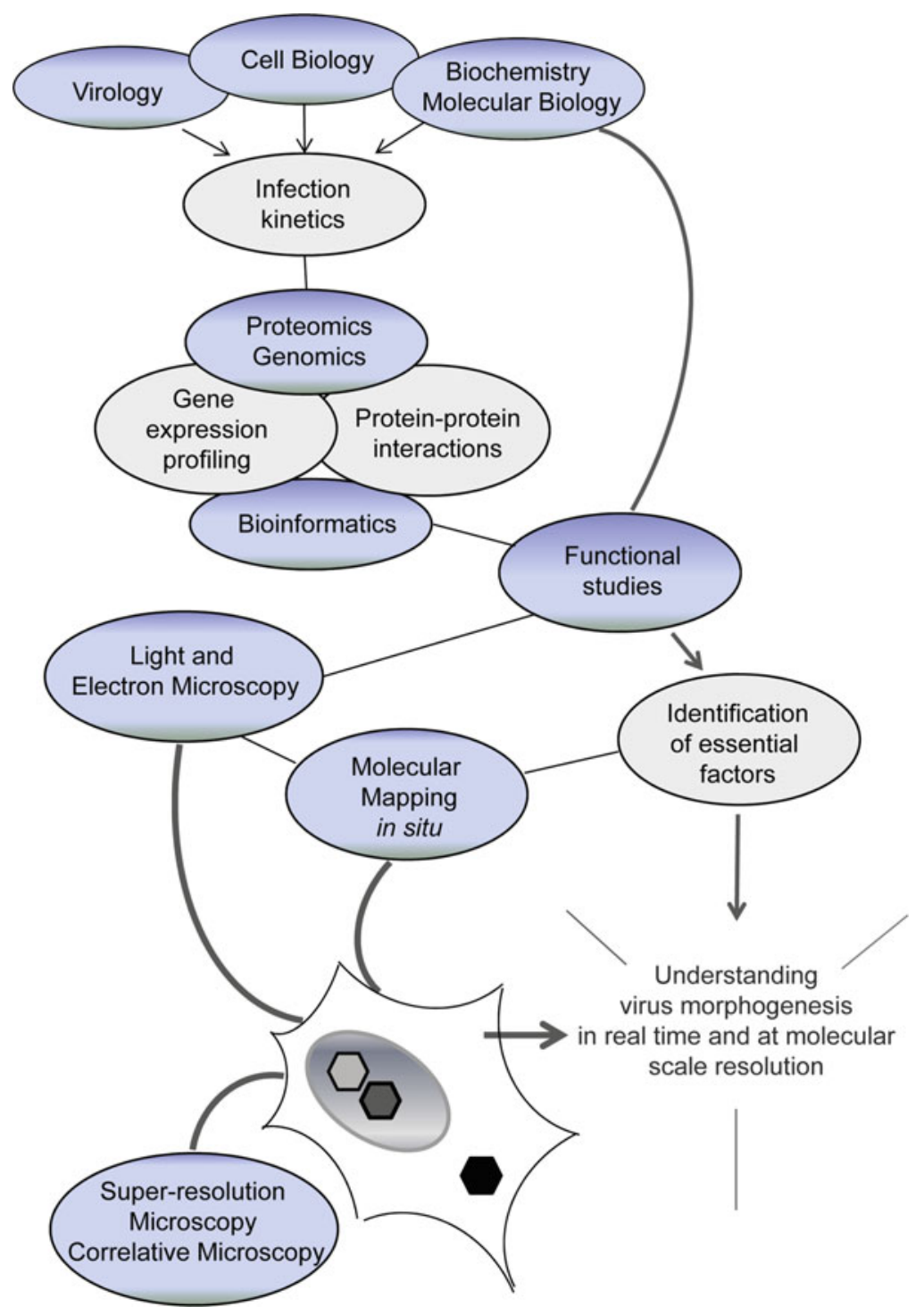

Fig. 14.8 Various technologies and their integration in the study of virus-cell interactions during viral morphogenesis. See text for a description

\section{References and Further Reading}

1. Novoa RR, Calderita G, Arranz R, Fontana J, Granzow H, Risco C (2005) Virus factories: associations of cell organelles for viral replication and morphogenesis. Biol Cell 97:147-172

2. Netherton CL, Wileman T (2011) Virus factories, double membrane vesicles and viroplasm generated in animal cells. Curr Opin Virol 1:381-387 
3. Nagy PD, Pogany J (2010) Global genomics and proteomics to identify host factors as targets to induce resistance against tomato bushy stunt virus. Adv Virus Res 76:123-177

4. Alkhalil A, Hammamieh R, Hardick J, Ichou MA, Jett M, Ibrahim S (2010) Gene expression profiling of monkeypox virus-infected cells reveals novel interfaces for host-virus interactions. Virol J 7:173

5. Friedel CC, Haas J (2011) Virus-host interactomes and global models of virus-infected cells. Trends Microbiol 19:501-508

6. Sessions OM, Barrows NJ, Souza-Neto JA, Robinson TJ, Hershey CL, Rodgers MA, Ramirez JL, Dimopoulos G, Yang PL, Pearson JL, Garcia-Blanco MA (2009) Discovery of insect and human dengue virus host factors. Nature 458:1047-1050

7. Meliopoulos VA, Andersen LE, Birrer KF, Simpson KJ, Lowenthal JW, Bean AG, Stambas J, Stewart CR, Tompkins SM, van Beusechem VW, Fraser I, Mhlanga M, Barichievy S, Smith Q, Leake D, Karpilow J, Buck A, Jona G, Tripp RA (2012) Host gene targets for novel influenza therapies elucidated by high-throughput RNA interference screens. FASEB J 26:1372-1386

8. Pache L, König R, Chanda SK (2010) Identifying HIV-1 host cell factors by genome-scale RNAi screening. Methods 53:3-12

9. Morita E, Sandrin V, McCullough J, Katsuyama A, Hamilton IB, Sundquist WI (2011) ESCRT-III protein requirements for HIV-1 budding. Cell Host Microbe 9:235-242

10. MacFarlane SA, Uhrig JF (2008) Yeast two-hybrid assay to identify host-virus interactions. Methods Mol Biol 451:649-672

11. De Chassey B, Navratil V, Tafforeau L, Hiet MS, Aublin-Gex A, Agaugué S, Meiffren G, Pradezynski F, Faria BF, Chantier T, Le Breton M, Pellet J, Davoust N, Mangeot PE, Chaboud A, Penin F, Jacob Y, Vidalain PO, Vidal M, André P, Raourdin-Combe C, Lotteau V (2008) Hepatitis C virus infection protein network. Mol Syst Biol 4:230

12. Zhang L, Villa NY, Rahman MM, Smallwood S, Shattuck D, Neff C, Dufford M, Lanchbury JS, Labaer J, McFadden G (2009) Analysis of vaccinia virus-host protein-protein interactions: validations of yeast two-hybrid screenings. J Proteome Res 8:4311-4318

13. König R, Zhou Y, Elleder D, Diamond TL, Bonamy GM, Ireland JT, Chiang CY, Tu BP, De Jesus PD, Lilley CE, Seidel S, Opaluch AM, Caldwell JS, Weitzman MD, Kuhen KL, Bandyopadhyay S, Ideker T, Orth AP, Miraglia LJ, Bushman FD, Young JA, Chanda SK (2008) Global analysis of host-pathogen interactions that regulate early-stage HIV-1 replication. Cell 135:49-60

14. Zhang B, Kraemer B, Sengupta D, Fields S, Wickens M (1999) Yeast three-hybrid system to detect and analyze interactions between RNA and protein. Methods Enzymol 306:93-113

15. Lee E-G, Linial ML (2004) Basic residues of the retroviral nucleocapsid play different roles in Gag-Gag and Gag-Z RNA interactions. J Virol 78:8486-8495

16. Jäger S, Gulbahce N, Cimermancic P, Kane J, He N, Chou S, D’Orso I, Fernandes J, Jan G, Frankel AD, Alber T, Zhou Q, Krogan NJ (2011) Purification and characterization of HIVhuman protein complexes. Methods 53:13-19

17. Zhang F, Zang T, Wilson SJ, Johnson MC, Bieniasz PD (2011) Clathrin facilitates the morphogenesis of retrovirus particles. PLoS Pathog 7:e1002119

18. Bartee E, McCormack A, Früh K (2006) Quantitative membrane proteomics reveals new cellular targets of viral immune modulators. PLoS Pathog 2:e107

19. Martin-Serrano J, Neil SJD (2011) Host factors involved in retroviral budding and release. Nat Rev Microbiol 9:519-531

20. Kuhl BJ, Cheng V, Wainberg MA, Liang C (2011) Tetherin and its viral antagonists. J Neuroimmune Pharmacol 6:188-201

21. Gonzalez O, Zimmer R (2011) Contextual analysis of RNAi-based functional screens using interaction networks. Bioinformatics 27:2707-2713

22. Fontana J, López-Montero N, Elliott RM, Fernández JJ, Risco C (2008) The unique architecture of bunyamwera virus factories around the Golgi complex. Cell Microbiol 10:2012-2028 
23. Katsafanas GC, Moss B (2007) Colocalization of transcription and translation within cytoplasmic poxvirus factories coordinates viral expression and subjugates host functions. Cell Host Microbe 2:221-228

24. Sivaraman D, Biswas P, Cella LN, Yates MV, Chen W (2011) Detecting RNA viruses in living mammalian cells by fluorescence microscopy. Trends Biotech 29:307-313

25. Bozzola JJ, Russell LD (1999) Electron microscopy. Principles and techniques for biologists. Jones and Bartlett Publishers, Inc, Boston

26. Goldsmith CS, Miller SE (2009) Modern uses of electron microscopy for detection of viruses. Clin Microbiol Rev 22:552-563

27. Fiala JC (2005) Reconstruct: a free editor for serial section microscopy. J Microsc 218:52-61

28. Schatten H (2011) Low-voltage high-resolution SEM (LVHRSEM) for biological structural and molecular analysis. Micron 42:175-185

29. Zauberman N, Mutsafi Y, Halevy DB, Shimoni E, Klein E, Xiao C, Sun S, Minsky A (2008) Distinct DNA exit and packaging portals in the virus Acanthamoeba polyphaga mimivirus. PLoS Biol 6:e114

30. Risco C, Rodríguez JR, López-Iglesias C, Carrascosa JL, Esteban M, Rodríguez D (2002) Endoplasmic reticulum-Golgi intermediate compartment membranes and vimentin filaments participate in vaccinia virus assembly. J Virol 76:1839-1855

31. Briggs JAG, Kräusslich H-G (2011) The molecular architecture of HIV. J Mol Biol 410:491-500

32. Welsch S, Miller S, Romero-Brey I, Merz A, Bleck CKE, Walther P, Fuller SD, Anthony C, Krijnse-Locker J, Bartenschlager R (2009) Composition and three-dimensional architecture of the dengue virus replication and assembly sites. Cell Host Microbe 5:365-375

33. Chlanda P, Carbajal MA, Cyrklaff M, Griffiths G, Krijnse-Locker J (2009) Membrane rupture generates single open membrane sheets during vaccinia virus assembly. Cell Host Microbe 6:81-90

34. Cyrklaff M, Risco C, Fernández JJ, Jiménez MV, Esteban M, Baumeister W, Carrascosa JL (2005) Cryo-electron tomography of vaccinia virus. Proc Natl Acad Sci USA 102:2772-2777

35. Kiernan JA (2008) Histological and histochemical methods. Theory and practice. Scion Publishing Ltd, Oxfordshire

36. Hayat MA (2002) Microscopy, immunohistochemistry, and antigen retrieval methods. Kluwer Academic//Plenum Publishers, New York

37. Van Weering JRT, Brown E, Sharp TH, Mantell J, Cullen PJ, Verkade P (2010) Intracellular membrane traffic at high resolution. Methods Cell Biol 96:619-648

38. Tokuyasu KT (1973) A technique for ultracryotomy of cell suspensions and tissues. J Cell Biol 57:551-565

39. Slot JW, Geuze HJ (2007) Cryosectioning and immunolabeling. Nat Protoc 2:2480-2491

40. Sodeik B, Doms RW, Ericsson M, Hiller G, Machamer CE, van't Hof W, van Meer G, Moss B, Griffiths G (1993) Assembly of vaccinia virus: role of the intermediate compartment between the endoplasmic reticulum and the Golgi stacks. J Cell Biol 121:521-541

41. Jouvenet N, Simon SM, Bieniasz PD (2011) Visualizing HIV-1 assembly. J Mol Biol 410:501-511

42. Shu X, Lev-Ram V, Deerinck TJ, Qi Y, Ramko EB, Davidson MW, Jin Y, Ellisman MH, Tsien RY (2011) A genetically encoded tag for correlated light and electron microscopy of intact cells, tissues and organisms. PLoS Biol 9:e1001041

43. Gaietta G, Deerinck TJ, Admas SR, Bouwer J, Tour O, Laird DW, Sosinsky GE, Tsien RY, Ellisman MH (2002) Multicolor and electron microscopic imaging of connexin trafficking. Science 296:503-507

44. La Scola B, Desnues C, Pagnier I, Robert C, Barrasi L, Fournous G, Merchat M, Suzan-Monti M, Forterre P, Koonin E, Raoul D (2008) The virophage as a unique parasite of the giant mimivirus. Nature 455:100-104

45. Carvalho ZG, Alves de Matos AP, Rodrigues-Pousada C (1988) Association of African swine fever virus with the cytoskeleton. Virus Res 11:175-192 
46. Rojo G, Chamorro M, Salas ML, Viñuela E, Cuezva JM, Salas J (1998) Migration of mitochondria to viral assembly sites in African Swine fever virus-infected cells. J Virol 72:7583-7588

47. Pierini R, Cottam E, Roberts R, Wileman T (2009) Modulation of membrane traffic between endoplasmic reticulum, ERGIC and Golgi to generate compartments for the replication of bacteria and viruses. Sem Cell Dev Biol 20:828-833

48. Fontana J, Tzeng WP, Calderita G, Fraile-Ramos A, Frey TK, Risco C (2007) Novel replication complex architecture in rubella replicon-transfected cells. Cell Microbiol 9:875-890

49. Deng Y, Almshwerqi ZA, Ng MML, Kohlwein SD (2010) Do viruses subvert cholesterol homeostasis to induce host cubic membranes? Trends Cell Biol 20:371-379

50. Liu STH, Sharon-Friling RS, Ivanova P, Milne SB, Myers DS, Rabinowitz JD, Brown HA, Shenk T (2011) Synaptic vesicle-like lipidome of human cytomegalovirus virions reveals a role for SNARE machinery in virion egress. Proc Natl Acad Sci USA 108:12869-12874

51. Harries P, Ding B (2011) Cellular factors in plant virus movement: at the leading edge of macromolecular trafficking in plants. Virology 411:237-243

52. Fontana J, López-Iglesias C, Tzeng WP, Frey TK, Fernández JJ, Risco C (2010) Threedimensional structure of rubella virus factories. Virology 405:579-591

53. Ilkow CS, Weckbecker D, Cho WJ, Meier S, Beatch MD, Goping IS, Herrmann JM, Hobman TC (2010) The rubella virus capsid protein inhibits mitochondrial import. J Virol 84:119-130

54. Huang B, Babcock H, Zhuang X (2011) Breaking the diffraction barrier: super-resolution imaging of cells. Cell 143:1047-1058

55. Jouvenet N, Zhadina M, Bieniasz PD, Simon SM (2011) Dynamics of ESCRT protein recruitment during retroviral assembly. Nat Cell Biol 13:394-401

56. Lehmann M, Rocha S, Mangeat B, Blanchet F, Uji-I H, Hofkens J, Piguet V (2011) Quantitative multicolor super-resolution microscopy reveals tetherin HIV-1 interaction. PLoS Pathog 7: e1002456

57. Monosov EZ, Wenzel TJ, Luers GH, Heyman JA, Subramani S (1996) Labeling of peroxisomes with green fluorescent protein in living $P$. pastoris cells. J Histochem Cytochem 44:581-589

58. Diestra E, Fontana J, Guichard P, Marco S, Risco C (2009) Visualization of proteins in intact cells with a clonable tag for electron microscopy. J Struct Biol 165:157-168

59. Wang Q, Mercogliano CP, Löwe J (2011) A ferritin-based label for cellular electron cryotomography. Structure 19:147-154

60. Risco C, Sanmartín-Conesa E, Tzeng WP, Frey TK, Seybold V, de Groot RJ (2012) Specific, sensitive, high-resolution detection of protein molecules in eukaryotic cells using metaltagging transmission electron microscopy. Structure 20:759-766

\section{Further Reading}

Baumgärtel V, Ivanchenko S, Dupont A, Sergeev M, Wiseman PW, Kräusslich HG, Bräuchle C, Müller B, Lamb DC (2011) Live-cell visualization of dynamics of HIV budding site interactions with an ESCRT component. Nat Cell Biol 13:469-474

Claverie JM, Abergel C (2009) Mimivirus and its virophage. Annu Rev Genet 43:49-66

Erickson KD, Bouchet-Marquis C, Heiser K, Szomolanyi-Tsuda E, Mishra R, Lamothe B, Hoenger A, Garcea RL (2012) Virion assembly factories in the nucleus of polyomavirusinfected cells. PLoS Pathog 8:e100263

Fogarty KH, Zhang W, Grigsby IF, Johnson JL, Chen Y, Mueller JD, Mansky LM (2011) New insights into HTLV-1 particle structure, assembly and Gag-Gag interactions in living cells. Viruses 3:770-793 
Fu C, Johnson J (2011) Viral life cycles captured in three-dimensions with electron microscopy tomography. Curr Opin Virol 1:125-133

Iwasaki K, Omura T (2010) Electron tomography of the supramolecular structure of virus-infected cells. Curr Opin Struct Biol 20:632-639

Mutsafi Y, Zauberman N, Sabanay O, Minsky A (2010) Vaccinia-like cytoplasmic replication of the giant mimivirus. Proc Natl Acad Sci USA 107:5978-5982

Nagy PD, Pogany J (2012) The dependence of viral RNA replication on co-opted host factors. Nat Rev Microbiol 10:137-149

Otto A, Bernhardt J, Hecker M, Becher D (2012) Global relative and absolute quantitation in microbial proteomics. Curr Opin Microbiol 15:1-9

Rust M, Lakadamyali M, Brandenburg B, Zhuang X (2008) Single-virus tracking in live cells. In: Selvin PR, Ha T (eds) Single molecule techniques: a laboratory manual. Cold Spring Harbor Laboratory Press, New York

Van Vliet K, Mohamed MR, Zhang L, Villa NY, Werden SJ, Liu J, McFadden G (2009) Poxvirus proteomics and virus-host protein interactions. Microbiol Mol Biol Rev 73:730-749

Also especially recommended for further reading are references $[1,2,3,5,19,37,49,54]$ listed above. 